Research Article

\title{
Strong Convergence of an Iterative Algorithm for Hierarchical Problems
}

\author{
Poom Kumam ${ }^{1}$ and Thanyarat Jitpeera ${ }^{2}$ \\ ${ }^{1}$ Department of Mathematics, Faculty of Science, King Mongkut's University of Technology Thonburi, Bang Mod, \\ Thung Khru, Bangkok 10140, Thailand \\ ${ }^{2}$ Department of Mathematics, Faculty of Science and Agriculture, Rajamangala University of Technology Lanna, \\ Phan, Chiangrai 57120, Thailand
}

Correspondence should be addressed to Thanyarat Jitpeera; t.jitpeera@hotmail.com

Received 26 April 2014; Revised 17 June 2014; Accepted 27 June 2014; Published 20 July 2014

Academic Editor: Wei-Shih Du

Copyright (C) 2014 P. Kumam and T. Jitpeera. This is an open access article distributed under the Creative Commons Attribution License, which permits unrestricted use, distribution, and reproduction in any medium, provided the original work is properly cited.

We introduce the triple hierarchical problem over the solution set of the variational inequality problem and the fixed point set of a nonexpansive mapping. The strong convergence of the algorithm is proved under some mild conditions. Our results extend those of Yao et al., Iiduka, Ceng et al., and other authors.

\section{Introduction}

Let $C$ be a closed convex subset of a real Hilbert space $H$ with inner product $\langle\cdot, \cdot\rangle$ and norm $\|\cdot\|$. We denote weak convergence and strong convergence by notations $\rightarrow$ and $\rightarrow$, respectively. Let $A$ be a nonlinear mapping. The HartmanStampacchia variational inequality [1] is to find $x \in C$ such that $\langle A x, y-x\rangle \geq 0, \forall y \in C$. The set of solutions is denoted by $\operatorname{VI}(C, A), f: C \rightarrow C$ is said to be a $\rho$-contraction if there exists a constant $\rho \in[0,1)$ such that $\|f(x)-f(y)\| \leq \rho \| x-$ $y \|, \forall x, y \in C$. A mapping $A: H \rightarrow H$ is said to be monotone if $\langle A x-A y, x-y\rangle \geq 0, \forall x, y \in H$. A mapping $A: H \rightarrow H$ is said to be $\alpha$-strongly monotone if there exists a positive real number $\alpha$ such that $\langle A x-A y, x-y\rangle \geq \alpha\|x-y\|^{2}, \forall x, y \in H$. A mapping $A: H \rightarrow H$ is said to be $\beta$-inverse-strongly monotone if there exists a positive real number $\beta$ such that $\langle A x-A y, x-y\rangle \geq \beta\|A x-A y\|^{2}, \forall x, y \in H$. A mapping $A: H \rightarrow H$ is said to be $L$-Lipschitz continuous if there exists a positive real number $L$ such that $\|A x-A y\| \leq L \| x-$ $y \|, \forall x, y \in H$. A linear bounded operator $A$ is said to be strongly positive on $H$ if there exists a constant $\bar{\gamma}>0$ with the property $\langle A x, x\rangle \geq \bar{\gamma}\|x\|^{2}, \forall x \in H$. A mapping $T: C \rightarrow C$ is said to be nonexpansive if $\|T x-T y\| \leq\|x-y\|, \forall x, y \in C$.

A point $x \in C$ is a fixed point of $T$ provided $T x=x$. Denote by $F(T)$ the set of fixed points of $T$; that is, $F(T)=$ $\{x \in C: T x=x\}$. If $C$ is bounded closed convex and $T$ is a nonexpansive mapping of $C$ into itself, then $F(T)$ is nonempty (see [2]).

We discuss the following variational inequality problem over the fixed point set of a nonexpansive mapping (see [3-16]), which is said to be the hierarchical problem. Let a monotone, continuous mapping $A: H \rightarrow H$ and a nonexpansive mapping $T: H \rightarrow H$. Find $x \in \operatorname{VI}(F(T), A)=$ $\{x \in F(T):\langle A x, y-x\rangle \geq 0, \forall y \in F(T)\}$, where $F(T) \neq \emptyset$. This solution set is denoted by $\Xi$.

We introduce the following variational inequality problem over the solution set of variational inequality problem and the fixed point set of a nonexpansive mapping (see [17, 18]), which is said to be the triple hierarchical problem. Let an inverse-strongly monotone $A: H \rightarrow H$, a strongly monotone and Lipschitz continuous $B: H \rightarrow H$, and a nonexpansive mapping $T: H \rightarrow H$. Find $x \in \operatorname{VI}(\Xi, B)=$ $\{x \in \Xi:\langle B x, y-x\rangle \geq 0, \forall y \in \Xi\}$, where $\Xi:=\operatorname{VI}(F(T), A) \neq$ $\emptyset$.

In 2009, Yao et al. [19] considered the following two-step iterative algorithm with the initial guess $x_{0} \in C$ which is chosen arbitrarily:

$$
\begin{aligned}
x_{n+1} & =\alpha_{n} f\left(x_{n}\right)+\left(1-\alpha_{n}\right) T y_{n}, \\
y_{n} & =\beta_{n} S x_{n}+\left(1-\beta_{n}\right) x_{n}, \quad \forall n \geq 0,
\end{aligned}
$$


where $\alpha_{n}, \beta_{n} \in(0,1)$ satisfies certain assumptions. Let $S, T$ be two nonexpansive mappings and let $f: C \rightarrow C$ be a contraction mapping. Then, they proved that the above iterative sequence $\left\{x_{n}\right\}$ converges strongly to fixed point.

Next, Iiduka [17] introduced a monotone variational inequality with variational inequality constraint over the fixed point set of a nonexpansive mapping; the sequence $\left\{x_{n}\right\}$ defined by the iterative method below, with the initial guess $x_{1} \in H$, is chosen arbitrarily:

$$
\begin{gathered}
y_{n}=T\left(x_{n}-\lambda_{n} A_{1} x_{n}\right), \\
x_{n+1}=y_{n}-\mu \alpha_{n} A_{2} y_{n}, \quad \forall n \geq 0,
\end{gathered}
$$

where $\alpha_{n} \in(0,1]$ and $\lambda_{n} \in(0,2 \alpha]$ satisfy certain conditions, $A_{1}: H \rightarrow H$ is an inverse-strongly monotone, $A_{2}$ : $H \rightarrow H$ is a strongly monotone and Lipschitz continuous, and $T: H \rightarrow H$ is a nonexpansive mapping; then the strongly convergence analysis of the sequence generated by (2) is proved under some appropriate conditions.

In 2011, Yao et al. [20] studied the hierarchical problem over the fixed point set. Let the sequences $\left\{x_{n}\right\}$ be generated by these two following algorithms:

implicit algorithm $x_{t}=T P_{C}[I-t(A-\gamma f)] x_{t}, \forall t \in$ $(0,1)$

explicit algorithm $x_{n+1}=\beta_{n} x_{n}+\left(1-\beta_{n}\right) T P_{C}\left[I-\alpha_{n}(A-\right.$ $\gamma f)] x_{n}, \forall n \geq 0$.

They illustrated that these two algorithms converge strongly to the unique solution of the variational inequality which is to find $x^{*} \in F(T)$ such that

$$
\left\langle(A-\gamma f) x^{*}, x-x^{*}\right\rangle \geq 0, \quad \forall x \in F(T),
$$

where $A: C \rightarrow H$ is a strongly positive linear bounded operator, $f: C \rightarrow H$ is a $\rho$-contraction, and $T: C \rightarrow C$ is a nonexpansive mapping satisfying some conditions.

Very recently, Ceng et al. [21] studied the following new algorithms. For $x_{0} \in C$ is chosen arbitrarily, they defined a sequence $\left\{x_{n}\right\}$ by

$$
\begin{array}{r}
x_{n+1} \\
=P_{C}\left[\lambda_{n} \gamma\left(\alpha_{n} f\left(x_{n}\right)+\left(1-\alpha_{n}\right) S x_{n}\right)+\left(I-\lambda_{n} \mu F\right) T x_{n}\right], \\
\forall n \geq 0,
\end{array}
$$

where the mappings $S, T$ are nonexpansive mappings with $F(T) \neq \emptyset$. Let $F: C \rightarrow H$ be a Lipschitzian and strongly monotone operator and let $f: C \rightarrow H$ be a contraction mapping satisfying some appropriate conditions. They proved that the proposed algorithms strongly converge to the minimum norm fixed point of $T$.

In this paper, we consider a new iterative algorithm for solving the triple hierarchical problem over the solution set of the variational inequality problem and the fixed point set of a nonexpansive mapping which contain algorithms (1) and (4) as follows:

$$
\begin{aligned}
y_{n} & =P_{C}\left[\beta_{n} S x_{n}+\left(1-\beta_{n}\right) x_{n}\right], \\
x_{n+1} & =\gamma \lambda_{n} \phi\left(x_{n}\right)+\left(I-\lambda_{n} \mu F\right) T y_{n}, \quad \forall n \geq 0,
\end{aligned}
$$

where the mappings $S, T$ are nonexpansive mappings with $F(T) \neq \emptyset$. Let $F: C \rightarrow H$ be a Lipschitzian and strongly monotone operator, and let $\phi: H \rightarrow H$ be a contraction mapping satisfying some mild conditions. Find a point $x^{*} \epsilon$ $F(T)$ such that

$$
\left\langle(I-S) x^{*}, x-x^{*}\right\rangle \geq 0, \quad \forall x \in F(T) .
$$

This solution set of (6) is denoted by $\Omega:=\operatorname{VI}(F(T), S)$. The strong convergence for the proposed algorithms to the solution is solved under some appropriate assumptions. Our results improve the results of Ceng et al. [21], Iiduka [17], Yao et al. [19], Yao et al. [20], and some authors.

\section{Preliminaries}

Let $C$ be a nonempty closed convex subset of $H$. There holds the following inequality in an inner product space $\|x+y\|^{2} \leq$ $\|x\|^{2}+2\langle y, x+y\rangle, \forall x, y \in H$. For every point $x \in H$, there exists a unique nearest point in $C$, denoted by $P_{C} x$, such that

$$
\left\|x-P_{C} x\right\| \leq\|x-y\|, \quad \forall y \in C .
$$

$P_{C}$ is called the metric projection of $H$ onto $C$. It is well known that $P_{C}$ is a nonexpansive mapping of $H$ onto $C$ and satisfies

$$
\left\langle x-y, P_{C} x-P_{C} y\right\rangle \geq\left\|P_{C} x-P_{C} y\right\|^{2},
$$

for every $x, y \in H$. Moreover, $P_{C} x$ is characterized by the following properties: $P_{C} x \in C$ and

$$
\begin{gathered}
\left\langle x-P_{C} x, y-P_{C} x\right\rangle \leq 0, \\
\|x-y\|^{2} \geq\left\|x-P_{C} x\right\|^{2}+\left\|y-P_{C} x\right\|^{2},
\end{gathered}
$$

for all $x \in H, y \in C$. Let $B$ be a monotone mapping of $C$ into $H$. In the context of the variational inequality problem the characterization of projection (9) implies the following:

$$
u \in V I(C, B) \Longleftrightarrow u=P_{C}(u-\lambda B u), \quad \lambda>0 .
$$

It is also known that $H$ satisfies the Opial's condition [22]; that is, for any sequence $\left\{x_{n}\right\} \subset H$ with $x_{n} \rightarrow x$, the inequality $\liminf _{n \rightarrow \infty}\left\|x_{n}-x\right\|<\liminf _{n \rightarrow \infty}\left\|x_{n}-y\right\|$ holds for every $y \in H$ with $x \neq y$.

Lemma 1 (see [23]). Let $C$ be a closed convex subset of a real Hilbert space $H$ and let $T: C \rightarrow C$ be a nonexpansive mapping. Then $I-T$ is demiclosed at zero; that is, $x_{n} \rightarrow$ $x$ and $x_{n}-T x_{n} \rightarrow 0$ imply $x=T x$.

Lemma 2 (see [24]). Let $\left\{x_{n}\right\}$ and $\left\{y_{n}\right\}$ be bounded sequences in a Banach space $X$ and let $\left\{\beta_{n}\right\}$ be a sequence in $[0,1]$ with $0<\liminf _{n \rightarrow \infty} \beta_{n} \leq \limsup _{n \rightarrow \infty} \beta_{n}<1$. Suppose $x_{n+1}=$ $\left(1-\beta_{n}\right) y_{n}+\beta_{n} x_{n}$ for all integers $n \geq 0$ and $\lim \sup _{n \rightarrow \infty}\left(\| y_{n+1}-\right.$ $\left.y_{n}\|-\| x_{n+1}-x_{n} \|\right) \leq 0$. Then, $\lim _{n \rightarrow \infty}\left\|y_{n}-x_{n}\right\|=0$.

Lemma 3 (see [10]). Let $B: H \rightarrow H$ be $\beta$-strongly monotone and L-Lipschitz continuous and let $\mu \in\left(0,2 \beta / L^{2}\right)$. For $\lambda \epsilon$ $[0,1]$, define $T_{\lambda}: H \rightarrow H$ by $T_{\lambda}(x):=x-\lambda \mu B(x)$ for all $x \in H$. Then, for all $x, y \in H,\left\|T_{\lambda}(x)-T_{\lambda}(y)\right\| \leq(1-\lambda \tau)\|x-y\|$ hold, where $\tau:=1-\sqrt{1-\mu\left(2 \beta-\mu L^{2}\right)} \in(0,1]$. 
Lemma 4 (see [25]). Assume that $\left\{a_{n}\right\}$ is a sequence of nonnegative real numbers such that

$$
a_{n+1} \leq\left(1-\gamma_{n}\right) a_{n}+\delta_{n}, \quad \forall n \geq 0
$$

where $\left\{\gamma_{n}\right\} \subset(0,1)$ and $\left\{\delta_{n}\right\}$ is a sequence in $\mathscr{R}$ such that

(i) $\sum_{n=1}^{\infty} \gamma_{n}=\infty$;

(ii) $\lim \sup _{n \rightarrow \infty}\left(\delta_{n} / \gamma_{n}\right) \leq 0$ or $\sum_{n=1}^{\infty}\left|\delta_{n}\right|<\infty$.

Then $\lim _{n \rightarrow \infty} a_{n}=0$.

\section{Strong Convergence Theorem}

In this section, we introduce an iterative algorithm of triple hierarchical for solving monotone variational inequality problems for $\kappa$-Lipschitzian and $\eta$-strongly monotone operators over the solution set of variational inequality problems and the fixed point set of a nonexpansive mapping.

Theorem 5. Let $C$ be a nonempty closed and convex subset of a real Hilbert space $H$. Let $F: C \rightarrow C$ be $\kappa$-Lipschitzian and $\eta$-strongly monotone operators with constant $\kappa$ and $\eta>0$, respectively, and let $\phi: C \rightarrow C$ be a $\rho$-contraction with coefficient $\rho \in[0,1)$. Let $T: C \rightarrow C$ be a nonexpansive mapping with $F(T) \neq \emptyset$, and let $S: H \rightarrow H$ be a nonexpansive mapping. Let $0<\mu<2 \eta / \kappa^{2}$ and $0<\gamma<\tau$, where $\tau=1-\sqrt{1-\mu\left(2 \eta-\mu \kappa^{2}\right)}$. Suppose that $\left\{x_{n}\right\}$ is a sequence generated by the following algorithm where $x_{0} \in C$ is chosen arbitrarily:

$$
\begin{aligned}
y_{n} & =P_{C}\left[\beta_{n} S x_{n}+\left(1-\beta_{n}\right) x_{n}\right], \\
x_{n+1} & =\gamma \lambda_{n} \phi\left(x_{n}\right)+\left(I-\lambda_{n} \mu F\right) T y_{n}, \quad \forall n \geq 0,
\end{aligned}
$$

where $\left\{\beta_{n}\right\},\left\{\lambda_{n}\right\}, \subset(0,1)$ satisfy the following conditions:

$$
\begin{aligned}
& \text { (C1): } \beta_{n} \leq k \lambda_{n} ; \\
& \text { (C2): } \lim _{n \rightarrow \infty} \lambda_{n}=0, \lim _{n \rightarrow \infty}\left(\left(\lambda_{n}-\lambda_{n-1}\right) / \lambda_{n}\right)=0 \\
& \sum_{n=0}^{\infty} \lambda_{n}=\infty ;
\end{aligned}
$$$$
\text { (C3): } \lim _{n \rightarrow \infty}\left(\left(\beta_{n}-\beta_{n-1}\right) / \beta_{n}\right)=0 \text {. }
$$

Then $\left\{x_{n}\right\}$ converges strongly to $x^{*} \in \Omega$, which is the unique solution of another variational inequality:

$$
\left\langle(\mu F-\gamma \phi) x^{*}, x-x^{*}\right\rangle \geq 0, \quad \forall x \in \Omega
$$

where $\Omega:=\operatorname{VI}(F(T), S) \neq \emptyset$.
Proof. We will divide the proof into four steps.

Step 1. We will show that $\left\{x_{n}\right\}$ is bounded. Indeed, for any $x^{*} \in F(T)$, we have

$$
\begin{aligned}
& \left\|y_{n}-x^{*}\right\| \\
& =\left\|P_{C}\left[\beta_{n} S x_{n}+\left(1-\beta_{n}\right) x_{n}\right]-P_{C} x^{*}\right\| \\
& \leq\left\|\beta_{n} S x_{n}+\left(1-\beta_{n}\right) x_{n}-x^{*}\right\| \\
& =\left\|\beta_{n}\left(S x_{n}-S x^{*}\right)+\left(1-\beta_{n}\right)\left(x_{n}-x^{*}\right)+\beta_{n}\left(S x^{*}-x^{*}\right)\right\| \\
& \leq \beta_{n}\left\|x_{n}-x^{*}\right\|+\left(1-\beta_{n}\right)\left\|x_{n}-x^{*}\right\|+\beta_{n}\left\|S x^{*}-x^{*}\right\| \\
& \leq\left\|x_{n}-x^{*}\right\|+\beta_{n}\left\|S x^{*}-x^{*}\right\| .
\end{aligned}
$$

From (13), we deduce that

$$
\begin{aligned}
& \left\|x_{n+1}-x^{*}\right\| \\
& =\left\|\gamma \lambda_{n} \phi\left(x_{n}\right)+\left(I-\lambda_{n} \mu F\right) T y_{n}-x^{*}\right\| \\
& =\| \gamma \lambda_{n}\left(\phi\left(x_{n}\right)-\phi\left(x^{*}\right)\right)+\left(I-\lambda_{n} \mu F\right)\left(T y_{n}-x^{*}\right) \\
& \quad+\lambda_{n}\left(\gamma \phi\left(x^{*}\right)-\mu F x^{*}\right) \| \\
& \leq \gamma \lambda_{n}\left\|\phi\left(x_{n}\right)-\phi\left(x^{*}\right)\right\|+\left(I-\lambda_{n} \mu F\right)\left\|T y_{n}-x^{*}\right\| \\
& \quad+\lambda_{n}\left\|\gamma \phi\left(x^{*}\right)-\mu F x^{*}\right\| \\
& \leq \gamma \rho \lambda_{n}\left\|x_{n}-x^{*}\right\|+\left(1-\lambda_{n} \tau\right)\left\|y_{n}-x^{*}\right\| \\
& \quad+\lambda_{n}\left\|\gamma \phi\left(x^{*}\right)-\mu F x^{*}\right\| .
\end{aligned}
$$

Substituting (15) into (16), we obtain

$$
\begin{aligned}
\left\|x_{n+1}-x^{*}\right\| & \\
\leq & \gamma \rho \lambda_{n}\left\|x_{n}-x^{*}\right\| \\
& +\left(1-\lambda_{n} \tau\right)\left\{\left\|x_{n}-x^{*}\right\|+\beta_{n}\left\|S x^{*}-x^{*}\right\|\right\} \\
& +\lambda_{n}\left\|\gamma \phi\left(x^{*}\right)-\mu F x^{*}\right\| \\
\leq & \gamma \rho \lambda_{n}\left\|x_{n}-x^{*}\right\|+\left(1-\lambda_{n} \tau\right)\left\|x_{n}-x^{*}\right\| \\
& +\beta_{n}\left\|S x^{*}-x^{*}\right\|+\lambda_{n}\left\|\gamma \phi\left(x^{*}\right)-\mu F x^{*}\right\| \\
\leq & {\left[1-\lambda_{n}(\tau-\gamma \rho)\right]\left\|x_{n}-x^{*}\right\|+k \lambda_{n}\left\|S x^{*}-x^{*}\right\| } \\
& +\lambda_{n}\left\|\gamma \phi\left(x^{*}\right)-\mu F x^{*}\right\| \\
\leq & {\left[1-\lambda_{n}(\tau-\gamma \rho)\right]\left\|x_{n}-x^{*}\right\| } \\
& +\lambda_{n}\left(k\left\|S x^{*}-x^{*}\right\|+\left\|\gamma \phi\left(x^{*}\right)-\mu F x^{*}\right\|\right) \\
\leq & \max \left\{\left\|x_{n}-x^{*}\right\|+\frac{1}{\tau-\gamma \rho}\right. \\
& \left.\times\left(k\left\|S x^{*}-x^{*}\right\|+\left\|\gamma \phi\left(x^{*}\right)-\mu F x^{*}\right\|\right)\right\} .
\end{aligned}
$$


By induction, it follows that

$$
\begin{aligned}
&\left\|x_{n}-x^{*}\right\| \\
& \leq \max \left\{\left\|x_{0}-x^{*}\right\|+\frac{1}{\tau-\gamma \rho}\right. \\
&\left.\times\left(k\left\|S x^{*}-x^{*}\right\|+\left\|\gamma \phi\left(x^{*}\right)-\mu F x^{*}\right\|\right)\right\}, \\
& n \geq 0 .
\end{aligned}
$$

Therefore, $\left\{x_{n}\right\}$ is bounded and so are $\left\{y_{n}\right\},\left\{T y_{n}\right\},\left\{S x_{n}\right\}$, $\left\{\phi\left(x_{n}\right)\right\}$, and $\left\{F T\left(y_{n}\right)\right\}$.

Step 2. We will show that $\lim _{n \rightarrow \infty}\left\|x_{n}-T x_{n}\right\|=0$. Setting $v_{n}:=\beta_{n} S x_{n}+\left(1-\beta_{n}\right) x_{n}$, we obtain

$$
\begin{aligned}
\| v_{n}- & v_{n-1} \| \\
= & \left\|\beta_{n} S x_{n}+\left(1-\beta_{n}\right) x_{n}-\beta_{n-1} S x_{n-1}-\left(1-\beta_{n-1}\right) x_{n-1}\right\| \\
= & \| \beta_{n}\left(S x_{n}-S x_{n-1}\right)+\left(\beta_{n}-\beta_{n-1}\right) S x_{n-1} \\
& \quad+\left(1-\beta_{n}\right)\left(x_{n}-x_{n-1}\right)+\left(\beta_{n-1}-\beta_{n}\right) x_{n-1} \| \\
\leq & \beta_{n}\left\|x_{n}-x_{n-1}\right\|+\left|\beta_{n}-\beta_{n-1}\right|\left(\left\|S x_{n-1}\right\|+\left\|x_{n-1}\right\|\right) \\
& +\left(1-\beta_{n}\right)\left\|x_{n}-x_{n-1}\right\| \\
\leq & \left\|x_{n}-x_{n-1}\right\|+\left|\beta_{n}-\beta_{n-1}\right|\left(\left\|S x_{n-1}\right\|+\left\|x_{n-1}\right\|\right),
\end{aligned}
$$

which implies that

$$
\begin{aligned}
\left\|y_{n}-y_{n-1}\right\| & =\left\|P_{C} v_{n}-P_{C} v_{n-1}\right\| \\
& \leq\left\|v_{n}-v_{n-1}\right\| \\
& \leq\left\|x_{n}-x_{n-1}\right\|+\left|\beta_{n}-\beta_{n-1}\right|\left(\left\|S x_{n-1}\right\|+\left\|x_{n-1}\right\|\right) .
\end{aligned}
$$

It follows from (13) that

$$
\begin{aligned}
& \left\|x_{n+1}-x_{n}\right\| \\
& =\| \gamma \lambda_{n} \phi\left(x_{n}\right)+\left(I-\lambda_{n} \mu F\right) T y_{n}-\gamma \lambda_{n-1} \phi\left(x_{n-1}\right) \\
& \quad-\left(I-\lambda_{n-1} \mu F\right) T y_{n-1} \| \\
& =\| \gamma \lambda_{n}\left(\phi\left(x_{n}\right)-\phi\left(x_{n-1}\right)\right)+\left(\lambda_{n}-\lambda_{n-1}\right) \gamma \phi\left(x_{n-1}\right) \\
& \quad+\left(I-\lambda_{n} \mu F\right) T y_{n}-\left(I-\lambda_{n-1} \mu F\right) T y_{n-1} \| \\
& \leq \gamma \rho \lambda_{n}\left\|x_{n}-x_{n-1}\right\|+\left|\lambda_{n}-\lambda_{n-1}\right| \gamma\left\|\phi\left(x_{n-1}\right)\right\| \\
& +\|\left(I-\lambda_{n} \mu F\right) T y_{n}-\left(I-\lambda_{n} \mu F\right) T y_{n-1} \\
& \quad+\left(I-\lambda_{n} \mu F\right) T y_{n-1}-\left(I-\lambda_{n-1} \mu F\right) T y_{n-1} \| \\
& \leq \gamma \rho \lambda_{n}\left\|x_{n}-x_{n-1}\right\|+\left|\lambda_{n}-\lambda_{n-1}\right| \gamma\left\|\phi\left(x_{n-1}\right)\right\| \\
& +\left(1-\lambda_{n} \tau\right)\left\|y_{n}-y_{n-1}\right\|+\left|\lambda_{n}-\lambda_{n-1}\right| \mu\left\|F T y_{n-1}\right\|
\end{aligned}
$$

$$
\begin{aligned}
\leq & \gamma \rho \lambda_{n}\left\|x_{n}-x_{n-1}\right\|+\left|\lambda_{n}-\lambda_{n-1}\right| \\
& \times\left(\gamma\left\|\phi\left(x_{n-1}\right)\right\|+\mu\left\|F T y_{n-1}\right\|\right) \\
& +\left(1-\lambda_{n} \tau\right)\left\{\left\|x_{n}-x_{n-1}\right\|+\left|\beta_{n}-\beta_{n-1}\right|\right. \\
& \left.\times\left(\left\|S x_{n-1}\right\|+\left\|x_{n-1}\right\|\right)\right\} \\
\leq & {\left[1-\lambda_{n}(\tau-\gamma \rho)\right]\left\|x_{n}-x_{n-1}\right\| } \\
& +\left|\lambda_{n}-\lambda_{n-1}\right|\left(\gamma\left\|\phi\left(x_{n-1}\right)\right\|+\mu\left\|F T y_{n-1}\right\|\right) \\
& +\left|\beta_{n}-\beta_{n-1}\right|\left(\left\|S x_{n-1}\right\|+\left\|x_{n-1}\right\|\right) \\
= & {\left[1-\lambda_{n}(\tau-\gamma \rho)\right]\left\|x_{n}-x_{n-1}\right\| } \\
& +\left(\frac{\left|\lambda_{n}-\lambda_{n-1}\right|}{\lambda_{n}}+\frac{\left|\beta_{n}-\beta_{n-1}\right|}{\lambda_{n}}\right) \lambda_{n} M_{1} \\
\leq & {\left[1-\lambda_{n}(\tau-\gamma \rho)\right]\left\|x_{n}-x_{n-1}\right\| } \\
& +\left(\frac{\left|\lambda_{n}-\lambda_{n-1}\right|}{\lambda_{n}}+\frac{k\left|\beta_{n}-\beta_{n-1}\right|}{\beta_{n}}\right) \lambda_{n} M_{1},
\end{aligned}
$$

where $M_{1}$ is a constant such that

$$
\sup _{n \geq 0}\left\{\left(\gamma\left\|\phi\left(x_{n}\right)\right\|+\mu\left\|F T y_{n}\right\|\right),\left(\left\|S x_{n}\right\|+\left\|x_{n}\right\|\right)\right\} \leq M_{1} \text {. }
$$

Hence, conditions (C2) and (C3) allow us to apply Lemma 4; then we get

$$
\lim _{n \rightarrow \infty}\left\|x_{n+1}-x_{n}\right\|=0
$$

By (21), we get

$$
\begin{aligned}
& \frac{\left\|x_{n+1}-x_{n}\right\|}{\lambda_{n}} \\
\leq & {\left[1-\lambda_{n}(\tau-\gamma \rho)\right] \frac{\left\|x_{n}-x_{n-1}\right\|}{\lambda_{n}} } \\
& +\frac{\left|\lambda_{n}-\lambda_{n-1}\right|+\left|\beta_{n}-\beta_{n-1}\right|}{\lambda_{n}} M_{1} \\
= & {\left[1-\lambda_{n}(\tau-\gamma \rho)\right] \frac{\left\|x_{n}-x_{n-1}\right\|}{\lambda_{n-1}} } \\
& +\left[1-\lambda_{n}(\tau-\gamma \rho)\right]\left(\frac{\left\|x_{n}-x_{n-1}\right\|}{\lambda_{n}}-\frac{\left\|x_{n}-x_{n-1}\right\|}{\lambda_{n-1}}\right) \\
& +\frac{\left|\lambda_{n}-\lambda_{n-1}\right|+\left|\beta_{n}-\beta_{n-1}\right|}{\lambda_{n}} M_{1} \\
\leq & {\left[1-\lambda_{n}(\tau-\gamma \rho)\right] \frac{\left\|x_{n}-x_{n-1}\right\|}{\lambda_{n-1}} } \\
& +M_{1} \lambda_{n} \frac{\left|\lambda_{n}-\lambda_{n-1}\right|+\left|\beta_{n}-\beta_{n-1}\right|}{\lambda_{n}^{2}} . \\
\lambda_{n-1} & \left|\frac{1}{\lambda_{n}}-\frac{1}{\lambda_{n-1}}\right|
\end{aligned}
$$


Using the conditions (C2) and (C3), we can apply Lemma 4 to conclude that

$$
\lim _{n \rightarrow \infty} \frac{\left\|x_{n+1}-x_{n}\right\|}{\lambda_{n}}=0 .
$$

By (13), we compute

$$
\begin{aligned}
\left\|x_{n+1}-T y_{n}\right\| & =\left\|\gamma \lambda_{n} \phi\left(x_{n}\right)+\left(I-\lambda_{n} \mu F\right) T y_{n}-T y_{n}\right\| \\
& =\left\|\gamma \lambda_{n} \phi\left(x_{n}\right)+T y_{n}-\lambda_{n} \mu F T y_{n}-T y_{n}\right\| \\
& \leq \lambda_{n}\left\|\gamma \phi\left(x_{n}\right)-\mu F T y_{n}\right\| .
\end{aligned}
$$

From the condition (C2), we note that $\lim _{n \rightarrow \infty}\left\|x_{n+1}-T y_{n}\right\|=$ 0 . At the same time, from (13), we also have

$$
\begin{aligned}
\left\|y_{n}-x_{n}\right\| & =\left\|P_{C}\left[\beta_{n} S x_{n}+\left(1-\beta_{n}\right) x_{n}\right]-P_{C} x_{n}\right\| \\
& \leq\left\|\beta_{n} S x_{n}+\left(1-\beta_{n}\right) x_{n}-x_{n}\right\| \\
& \leq \beta_{n}\left\|S x_{n}-x_{n}\right\| .
\end{aligned}
$$

By the conditions (C1) and (C2), we note that $\lim _{n \rightarrow \infty} \| y_{n}-$ $x_{n} \|=0$. Consider

$$
\begin{aligned}
\left\|y_{n}-T y_{n}\right\| \leq & \left\|y_{n}-x_{n}\right\|+\left\|x_{n}-x_{n+1}\right\| \\
& +\left\|x_{n+1}-T y_{n}\right\| \longrightarrow 0 .
\end{aligned}
$$

From (23), (26), and (27), we obtain

$$
\lim _{n \rightarrow \infty}\left\|y_{n}-T y_{n}\right\|=0 \text {. }
$$

We set $v_{n}=\beta_{n} S x_{n}+\left(1-\beta_{n}\right) x_{n}$; then we get

$$
\begin{aligned}
\left\|y_{n}-v_{n}\right\| & =\left\|P_{C} v_{n}-v_{n}\right\| \\
& \leq\left\|v_{n}-v_{n}\right\| \longrightarrow 0, \quad \text { as } n \longrightarrow \infty .
\end{aligned}
$$

From (13), we have

$$
\begin{aligned}
\left\|T y_{n}-T x_{n}\right\| & =\left\|T P_{C}\left[\beta_{n} S x_{n}+\left(1-\beta_{n}\right) x_{n}\right]-T P_{C} x_{n}\right\| \\
& \leq\left\|\beta_{n} S x_{n}+\left(1-\beta_{n}\right) x_{n}-x_{n}\right\| \\
& \leq \beta_{n}\left\|S x_{n}-x_{n}\right\| .
\end{aligned}
$$

By the conditions (C1) and (C2) again, we note that $\lim _{n \rightarrow \infty}\left\|T y_{n}-T x_{n}\right\|=0$. Consider

$$
\left\|x_{n}-T x_{n}\right\| \leq\left\|x_{n}-y_{n}\right\|+\left\|y_{n}-T y_{n}\right\|+\left\|T y_{n}-T x_{n}\right\| \longrightarrow 0 \text {. }
$$

From (29), $\lim _{n \rightarrow \infty}\left\|x_{n}-y_{n}\right\|=0$, and $\lim _{n \rightarrow \infty}\left\|T y_{n}-T x_{n}\right\|=$ 0 , we obtain

$$
\lim _{n \rightarrow \infty}\left\|x_{n}-T x_{n}\right\|=0
$$

Step 3. We will show that $\lim \sup _{n \rightarrow \infty}\left\langle\mu F x^{*}-\gamma \phi\left(x^{*}\right), x_{n}-\right.$ $\left.x^{*}\right\rangle \leq 0$. Rewrite (13) as

$$
\begin{aligned}
x_{n+1}= & \gamma \lambda_{n} \phi\left(x_{n}\right)+\left(I-\mu \lambda_{n} F\right) T y_{n} \\
& -v_{n}+\beta_{n} S x_{n}+\left(1-\beta_{n}\right) x_{n} .
\end{aligned}
$$

We observe that

$$
\begin{aligned}
x_{n} & -x_{n+1} \\
= & x_{n}-\gamma \lambda_{n} \phi\left(x_{n}\right) \\
& -\left(I-\mu \lambda_{n} F\right) T y_{n}+v_{n}-\beta_{n} S x_{n}-x_{n}+\beta_{n} x_{n} \\
= & \lambda_{n}(\mu F-\gamma \phi) x_{n} \\
& -\lambda_{n} \mu F x_{n}-\left(I-\mu \lambda_{n} F\right) T y_{n}+\left(I-\mu \lambda_{n} F\right) y_{n} \\
& -\left(I-\mu \lambda_{n} F\right) y_{n}+v_{n}+\beta_{n}(I-S) x_{n} \\
= & \lambda_{n}(\mu F-\gamma \phi) x_{n}+\lambda_{n} \mu\left(F y_{n}-F x_{n}\right)+\left(y_{n}-T y_{n}\right) \\
& -\mu \lambda_{n} F\left(y_{n}-T y_{n}\right)+\left(v_{n}-y_{n}\right)+\beta_{n}(I-S) x_{n} \\
= & \lambda_{n}(\mu F-\gamma \phi) x_{n}+\lambda_{n} \mu\left(F y_{n}-F x_{n}\right)+\left(y_{n}-T y_{n}\right) \\
& -\mu \lambda_{n} F\left(y_{n}-T y_{n}\right)+\lambda_{n}\left(y_{n}-T y_{n}\right) \\
& -\lambda_{n}\left(y_{n}-T y_{n}\right)+\left(v_{n}-y_{n}\right)+\beta_{n}(I-S) x_{n} \\
= & \lambda_{n}(\mu F-\gamma \phi) x_{n}+\lambda_{n} \mu\left(F y_{n}-F x_{n}\right) \\
& +\lambda_{n}(I-\mu F)\left(y_{n}-T y_{n}\right)+\left(1-\lambda_{n}\right)\left(y_{n}-T y_{n}\right) \\
& +\left(v_{n}-y_{n}\right)+\beta_{n}(I-S) x_{n} .
\end{aligned}
$$

Set

$$
z_{n}=\frac{x_{n}-x_{n+1}}{\lambda_{n}}, \quad \forall n \geq 0 .
$$

We note from (35) that

$$
\begin{aligned}
z_{n}= & (\mu F-\gamma \phi) x_{n}+\mu\left(F y_{n}-F x_{n}\right)+(I-\mu F)\left(y_{n}-T y_{n}\right) \\
& +\frac{1-\lambda_{n}}{\lambda_{n}}\left(y_{n}-T y_{n}\right) \\
& +\frac{1}{\lambda_{n}}\left(v_{n}-y_{n}\right)+\frac{\beta_{n}}{\lambda_{n}}(I-S) x_{n} .
\end{aligned}
$$

This yields that, for each $x^{*} \in F(T)$,

$$
\begin{aligned}
& \left\langle z_{n}, x_{n}-x^{*}\right\rangle \\
& =\left\langle(\mu F-\gamma \phi) x_{n}, x_{n}-x^{*}\right\rangle+\mu\left\langle\left(F y_{n}-F x_{n}\right), x_{n}-x^{*}\right\rangle \\
& \quad+\left\langle(I-\mu F) y_{n}-(I-\mu F) T y_{n}, x_{n}-x^{*}\right\rangle \\
& \quad+\frac{1-\lambda_{n}}{\lambda_{n}}\left\langle y_{n}-T y_{n}, x_{n}-x^{*}\right\rangle \\
& \quad+\frac{1}{\lambda_{n}}\left\langle v_{n}-y_{n}, x_{n}-x^{*}\right\rangle+\frac{\beta_{n}}{\lambda_{n}}\left\langle(I-S) x_{n}, x_{n}-x^{*}\right\rangle
\end{aligned}
$$




$$
\begin{aligned}
= & \left\langle(\mu F-\gamma \phi) x^{*}, x_{n}-x^{*}\right\rangle \\
& +\left\langle(\mu F-\gamma \phi) x_{n}-(\mu F-\gamma \phi) x^{*}, x_{n}-x^{*}\right\rangle \\
& +\mu\left\langle\left(F y_{n}-F x_{n}\right), x_{n}-x^{*}\right\rangle \\
& +\left\langle(I-\mu F) y_{n}-(I-\mu F) T y_{n}, x_{n}-x^{*}\right\rangle \\
& +\frac{1-\lambda_{n}}{\lambda_{n}}\left\langle y_{n}-T y_{n}, x_{n}-x^{*}\right\rangle+\frac{1}{\lambda_{n}}\left\langle v_{n}-y_{n}, x_{n}-x^{*}\right\rangle \\
& +\frac{\beta_{n}}{\lambda_{n}}\left\langle(I-S) x_{n}, x_{n}-x^{*}\right\rangle .
\end{aligned}
$$

In view of (38), $\left\langle(\mu F-\gamma \phi) x_{n}-(\mu F-\gamma \phi) x^{*}, x_{n}-x^{*}\right\rangle$ is nonnegative due to the monotonicity of $\mu F-\gamma \phi$. From (38), we derive that

$$
\begin{aligned}
\left\langle z_{n}, x_{n}-x^{*}\right\rangle \geq & \left\langle(\mu F-\gamma \phi) x^{*}, x_{n}-x^{*}\right\rangle \\
& +\mu\left\langle\left(F y_{n}-F x_{n}\right), x_{n}-x^{*}\right\rangle \\
& +\left\langle(I-\mu F) y_{n}-(I-\mu F) T y_{n}, x_{n}-x^{*}\right\rangle \\
& +\frac{1-\lambda_{n}}{\lambda_{n}}\left\langle y_{n}-T y_{n}, x_{n}-x^{*}\right\rangle \\
& +\frac{1}{\lambda_{n}}\left\langle v_{n}-y_{n}, x_{n}-x^{*}\right\rangle \\
& +\frac{\beta_{n}}{\lambda_{n}}\left\langle(I-S) x_{n}, x_{n}-x^{*}\right\rangle .
\end{aligned}
$$

Since (29) implies $\left\|(I-\mu F) y_{n}-(I-\mu F) T y_{n}\right\| \rightarrow 0$, as $n \rightarrow \infty$, from (25), then we get $z_{n} \rightarrow 0$. Using (C1) and (30), $\| y_{n}-$ $x_{n} \| \rightarrow 0$, as $n \rightarrow \infty$ and $\left\{x_{n}\right\}$ is bounded. We obtain from (39) that

$$
\limsup _{n \rightarrow \infty}\left\langle(\mu F-\gamma \phi) x^{*}, x_{n}-x^{*}\right\rangle \leq 0, \quad \forall x^{*} \in F(T)
$$

Since the sequence $\left\{x_{n}\right\}$ is bounded, we can take a subsequence $\left\{x_{n_{j}}\right\}$ of $\left\{x_{n}\right\}$ such that

$$
\begin{aligned}
& \limsup _{n \rightarrow \infty}\left\langle(\mu F-\gamma \phi) x^{*}, x_{n}-x^{*}\right\rangle \\
& \quad=\limsup _{j \rightarrow \infty}\left\langle(\mu F-\gamma \phi) x^{*}, x_{n_{j}}-x^{*}\right\rangle
\end{aligned}
$$

and $x_{n_{j}} \rightarrow \tilde{x}$. From (33), by the demiclosed principle of the nonexpansive mapping, it follows that $\tilde{x} \in F(T)$. Then

$$
\begin{gathered}
\limsup _{j \rightarrow \infty}\left\langle(\mu F-\gamma \phi) x^{*}, x_{n_{j}}-x^{*}\right\rangle \\
\quad=\left\langle(\mu F-\gamma \phi) x^{*}, \tilde{x}-x^{*}\right\rangle \leq 0 .
\end{gathered}
$$

Step 4. Finally, we will prove $x_{n+1} \rightarrow x^{*}$. From (13), we note that

$$
\begin{aligned}
\left\|y_{n}-x^{*}\right\|^{2}= & \left\|P_{C}\left[\beta_{n} S x_{n}+\left(1-\beta_{n}\right) x_{n}\right]-P_{C} x^{*}\right\|^{2} \\
\leq & \left\|\left[\beta_{n} S x_{n}+\left(1-\beta_{n}\right) x_{n}\right]-x^{*}\right\|^{2} \\
\leq & \| \beta_{n}\left(S x_{n}-S x^{*}\right)+\left(1-\beta_{n}\right)\left(x_{n}-x^{*}\right) \\
& +\beta_{n}\left(S x^{*}-x^{*}\right) \|^{2} \\
\leq & \left\|\beta_{n}\left(S x_{n}-S x^{*}\right)+\left(1-\beta_{n}\right)\left(x_{n}-x^{*}\right)\right\|^{2} \\
& +2 \beta_{n}\left\langle S x^{*}-x^{*}, y_{n}-x^{*}\right\rangle \\
\leq & \beta_{n}\left\|x_{n}-x^{*}\right\|^{2}+\left(1-\beta_{n}\right)\left\|x_{n}-x^{*}\right\|^{2} \\
& +2 \beta_{n}\left\langle S x^{*}-x^{*}, y_{n}-x^{*}\right\rangle \\
\leq & \left\|x_{n}-x^{*}\right\|^{2}+2 \beta_{n}\left\|S x^{*}-x^{*}\right\|\left\|y_{n}-x^{*}\right\| .
\end{aligned}
$$

Using (43), we compute

$$
\begin{aligned}
& \left\|x_{n+1}-x^{*}\right\|^{2} \\
& =\left\|\gamma \lambda_{n} \phi\left(x_{n}\right)+\left(I-\lambda_{n} \mu F\right) T y_{n}-x^{*}\right\|^{2} \\
& =\| \gamma \lambda_{n}\left(\phi\left(x_{n}\right)-\phi\left(x^{*}\right)\right) \\
& +\left(I-\lambda_{n} \mu F\right) T y_{n}-\left(I-\lambda_{n} \mu F\right) x^{*} \\
& +\left(I-\lambda_{n} \mu F\right) x^{*}-x^{*}+\gamma \lambda_{n} \phi\left(x^{*}\right) \|^{2} \\
& =\| \gamma \lambda_{n}\left(\phi\left(x_{n}\right)-\phi\left(x^{*}\right)\right)+\left(I-\lambda_{n} \mu F\right)\left(T y_{n}-x^{*}\right) \\
& +\lambda_{n}\left(\gamma \phi\left(x^{*}\right)-\mu F x^{*}\right) \|^{2} \\
& \leq\left\|\gamma \lambda_{n}\left(\phi\left(x_{n}\right)-\phi\left(x^{*}\right)\right)+\left(I-\lambda_{n} \mu F\right)\left(T y_{n}-x^{*}\right)\right\|^{2} \\
& +2 \lambda_{n}\left\langle\gamma \phi\left(x^{*}\right)-\mu F x^{*}, x_{n+1}-x^{*}\right\rangle \\
& \leq \gamma^{2} \lambda_{n}^{2}\left\|\phi\left(x_{n}\right)-\phi\left(x^{*}\right)\right\|^{2}+\left(1-\lambda_{n} \tau\right)^{2}\left\|T y_{n}-x^{*}\right\|^{2} \\
& +2 \lambda_{n}\left\langle\gamma \phi\left(x^{*}\right)-\mu F x^{*}, x_{n+1}-x^{*}\right\rangle \\
& +2\left\langle\gamma \lambda_{n}\left(\phi\left(x_{n}\right)-\phi\left(x^{*}\right)\right),\left(I-\mu \lambda_{n} F\right)\left(T y_{n}-x^{*}\right)\right\rangle \\
& \leq \gamma^{2} \rho^{2} \lambda_{n}^{2}\left\|x_{n}-x^{*}\right\|^{2}+\left(1-2 \lambda_{n} \tau+\lambda_{n}^{2} \tau^{2}\right)\left\|y_{n}-x^{*}\right\|^{2} \\
& +2 \lambda_{n}\left\langle\gamma \phi\left(x^{*}\right)-\mu F x^{*}, x_{n+1}-x^{*}\right\rangle \\
& +2 \gamma \lambda_{n}\left\langle\phi\left(x_{n}\right)-\phi\left(x^{*}\right),\left(I-\mu \lambda_{n} F\right) T y_{n}-\left(I-\mu \lambda_{n} F\right) x^{*}\right\rangle \\
& =\gamma^{2} \rho^{2} \lambda_{n}^{2}\left\|x_{n}-x^{*}\right\|^{2}+\left(1-2 \lambda_{n} \tau+\lambda_{n}^{2} \tau^{2}\right)\left\|y_{n}-x^{*}\right\|^{2} \\
& +2 \lambda_{n}\left\langle\gamma \phi\left(x^{*}\right)-\mu F x^{*}, x_{n+1}-x^{*}\right\rangle \\
& +2 \gamma \lambda_{n}\left\langle\phi\left(x_{n}\right)-\phi\left(x^{*}\right),\left(T y_{n}-x^{*}\right)-\mu \lambda_{n} F\left(T y_{n}-x^{*}\right)\right\rangle
\end{aligned}
$$




$$
\begin{aligned}
= & \gamma^{2} \rho^{2} \lambda_{n}^{2}\left\|x_{n}-x^{*}\right\|^{2}+\left(1-2 \lambda_{n} \tau+\lambda_{n}^{2} \tau^{2}\right)\left\|y_{n}-x^{*}\right\|^{2} \\
& +2 \lambda_{n}\left\langle\gamma \phi\left(x^{*}\right)-\mu F x^{*}, x_{n+1}-x^{*}\right\rangle \\
& +2 \gamma \lambda_{n}\left\langle\phi\left(x_{n}\right)-\phi\left(x^{*}\right), T y_{n}-x^{*}\right\rangle \\
& -2 \gamma \lambda_{n}\left\langle\phi\left(x_{n}\right)-\phi\left(x^{*}\right), \mu \lambda_{n} F\left(T y_{n}-x^{*}\right)\right\rangle \\
\leq & \gamma^{2} \rho^{2} \lambda_{n}^{2}\left\|x_{n}-x^{*}\right\|^{2}+\left(1-2 \lambda_{n} \tau+\lambda_{n}^{2} \tau^{2}\right) \\
& +\left\{\left\|x_{n}-x^{*}\right\|^{2}+2 \beta_{n}\left\|S x^{*}-x^{*}\right\|\left\|y_{n}-x^{*}\right\|\right\} \\
& +2 \lambda_{n}\left\langle\gamma \phi\left(x^{*}\right)-\mu F x^{*}, x_{n+1}-x^{*}\right\rangle \\
& +2 \gamma \rho \lambda_{n}\left\|x_{n}-x^{*}\right\|\left\|T y_{n}-x^{*}\right\| \\
& -2 \gamma \rho \mu \lambda_{n}^{2}\left\|x_{n}-x^{*}\right\|\left\|F\left(T y_{n}-x^{*}\right)\right\| \\
\leq & {\left[1-\lambda_{n}\left(2 \tau-\lambda_{n} \tau^{2}-\lambda_{n} \gamma^{2} \rho^{2}\right)\right]\left\|x_{n}-x^{*}\right\|^{2} } \\
& +2 \varepsilon_{n} \lambda_{n}\left\|S x^{*}-x^{*}\right\|\left\|y_{n}-x^{*}\right\| \\
& +2 \lambda_{n}\left\langle\gamma \phi\left(x^{*}\right)-\mu F x^{*}, x_{n+1}-x^{*}\right\rangle \\
& +2 \gamma \rho \lambda_{n}\left\|x_{n}-x^{*}\right\|\left\|T y_{n}-x^{*}\right\| \\
& -2 \gamma \rho \mu \lambda_{n}^{2}\left\|x_{n}-x^{*}\right\|\left\|F\left(T y_{n}-x^{*}\right)\right\| .
\end{aligned}
$$

Since $\left\{x_{n}\right\},\left\{T y_{n}\right\}$, and $\left\{F T y_{n}\right\}$ are all bounded, we can choose a constant $M_{2}>0$ such that

$$
\begin{aligned}
& \sup _{n \geq 0} \frac{1}{2 \tau-\lambda_{n} \tau^{2}-\lambda_{n} \gamma^{2} \rho^{2}} \\
& \quad \times\left\{2 \gamma \rho \mu\left\|x_{n}-x^{*}\right\|\left\|F\left(T y_{n}-x^{*}\right)\right\|\right\} \leq M_{2} .
\end{aligned}
$$

It follows that

$$
\begin{aligned}
\left\|x_{n+1}-x^{*}\right\|^{2} \leq & {\left[1-\lambda_{n}\left(2 \tau-\lambda_{n} \tau^{2}-\lambda_{n} \gamma^{2} \rho^{2}\right)\right]\left\|x_{n}-x^{*}\right\|^{2} } \\
& +\lambda_{n}\left(2 \tau-\lambda_{n} \tau^{2}-\lambda_{n} \gamma^{2} \rho^{2}\right) \delta_{n},
\end{aligned}
$$

where

$$
\begin{aligned}
\delta_{n}= & \frac{2 \varepsilon_{n}}{2 \tau-\lambda_{n} \tau^{2}-\lambda_{n} \gamma^{2} \rho^{2}}\left\|S x^{*}-x^{*}\right\|\left\|y_{n}-x^{*}\right\| \\
& +\frac{2}{2 \tau-\lambda_{n} \tau^{2}-\lambda_{n} \gamma^{2} \rho^{2}}\left\langle\gamma \phi\left(x^{*}\right)-\mu F x^{*}, x_{n+1}-x^{*}\right\rangle \\
& +\frac{2}{2 \tau-\lambda_{n} \tau^{2}-\lambda_{n} \gamma^{2} \rho^{2}} \gamma \rho\left\|x_{n}-x^{*}\right\|\left\|T y_{n}-x^{*}\right\| \\
& -\lambda_{n} M_{2} .
\end{aligned}
$$

Now, applying Lemma 4 and (35), we conclude that $x_{n} \rightarrow$ $x^{*}$. This completes the proof.

Corollary 6. Let $C$ be a nonempty closed and convex subset of a real Hilbert space $H$. Let $F: C \rightarrow C$ be $\kappa$-Lipschitzian and $\eta$-strongly monotone operators with constant $\kappa$ and $\eta>0$, respectively. Let $T: C \rightarrow C$ be a nonexpansive mapping with $F(T) \neq \emptyset$, and let $S: H \rightarrow H$ be a nonexpansive mapping. Let $0<\mu<2 \eta / \kappa^{2}$ and $0<\gamma<\tau$, where $\tau=1-\sqrt{1-\mu\left(2 \eta-\mu \kappa^{2}\right)}$. Suppose $\left\{x_{n}\right\}$ is a sequence generated by the following algorithm $x_{0} \in C$ arbitrarily:

$$
x_{n+1}=\left(I-\lambda_{n} \mu F\right) T P_{C}\left[\beta_{n} S x_{n}+\left(1-\beta_{n}\right) x_{n}\right], \quad \forall n \geq 0,
$$

where $\left\{\beta_{n}\right\},\left\{\lambda_{n}\right\} \subset(0,1)$ satisfy the following conditions (C1)(C3). Then $\left\{x_{n}\right\}$ converges strongly to $x^{*} \in \Omega$, which is the unique solution of variational inequality:

$$
\left\langle(I-\mu F) x^{*}, x-x^{*}\right\rangle \geq 0, \quad \forall x \in \Omega,
$$

where $\Omega:=V I(F(T), S) \neq \emptyset$.

Proof. Putting $\phi \equiv 0$ in Theorem 5, we can obtain the desired conclusion immediately.

Corollary 7. Let $C$ be a nonempty closed and convex subset of a real Hilbert space $H$. Let $\phi: H \rightarrow H$ be a $\rho$-contraction with coefficient $\rho \in[0,1)$, and let $T: C \rightarrow C$ be a nonexpansive mapping with $F(T) \neq \emptyset$ and $S: H \rightarrow H$ a nonexpansive mapping. Suppose $\left\{x_{n}\right\}$ is a sequence generated by the following algorithm, $x_{0} \in C$, arbitrarily:

$$
\begin{gathered}
y_{n}=P_{C}\left[\beta_{n} S x_{n}+\left(1-\beta_{n}\right) x_{n}\right], \\
x_{n+1}=\lambda_{n} \phi\left(x_{n}\right)+\left(1-\lambda_{n}\right) T y_{n}, \quad \forall n \geq 0,
\end{gathered}
$$

where $\left\{\beta_{n}\right\},\left\{\lambda_{n}\right\} \subset(0,1)$ satisfy the following conditions (C1)(C3). Then $\left\{x_{n}\right\}$ converges strongly to $x^{*} \in \Omega$, which is the unique solution of variational inequality:

$$
\left\langle(I-\phi) x^{*}, x-x^{*}\right\rangle \geq 0, \quad \forall x \in \Omega,
$$

where $\Omega:=V I(F(T), S) \neq \emptyset$.

Proof. Putting $\gamma=1, \mu=2$, and $F \equiv I / 2$ in Theorem 5, we can obtain the desired conclusion immediately.

Corollary 8. Let $C$ be a nonempty closed and convex subset of a real Hilbert space $H$. Let $T: C \rightarrow C$ be a nonexpansive mapping with $F(T) \neq \emptyset$ and let $S: H \rightarrow H$ be a nonexpansive mapping. Suppose $\left\{x_{n}\right\}$ is a sequence generated by the following algorithm, $x_{0} \in C$, arbitrarily:

$$
x_{n+1}=\left(1-\lambda_{n}\right) T P_{C}\left[\beta_{n} S x_{n}+\left(1-\beta_{n}\right) x_{n}\right], \quad \forall n \geq 0,
$$

where $\left\{\beta_{n}\right\},\left\{\lambda_{n}\right\} \subset(0,1)$ satisfy the following conditions (C1)(C3). Then $\left\{x_{n}\right\}$ converges strongly to $x^{*} \in F(T)$, which is the unique solution of variational inequality:

$$
\left\langle(I-S) x^{*}, x-x^{*}\right\rangle \geq 0, \quad \forall x \in F(T) .
$$

Proof. Putting $\phi \equiv 0$ in Corollary 7, we can obtain the desired conclusion immediately. 
Corollary 9. Let $C$ be a nonempty closed and convex subset of a real Hilbert space $H$. Let $\phi: H \rightarrow H$ be a $\rho$-contraction with coefficient $\rho \in[0,1)$, and let $T: C \rightarrow C$ be a nonexpansive mapping with $F(T) \neq \emptyset$ and $S: C \rightarrow C$ a nonexpansive mapping. Suppose $\left\{x_{n}\right\}$ is a sequence generated by the following algorithm, $x_{0} \in C$, arbitrarily:

$$
\begin{array}{r}
x_{n+1}=\lambda_{n} x_{n}+\left(1-\lambda_{n}\right) T\left[\beta_{n} S x_{n}+\left(1-\beta_{n}\right) x_{n}\right], \\
\forall n \geq 0,
\end{array}
$$

where $\left\{\beta_{n}\right\},\left\{\lambda_{n}\right\} \subset(0,1)$ satisfy the following conditions $(C 1)$ (C3). Then $\left\{x_{n}\right\}$ converges strongly to $x^{*} \in F(T)$, which is the unique solution of variational inequality:

$$
\left\langle(I-S) x^{*}, x-x^{*}\right\rangle \geq 0, \quad \forall x \in F(T) .
$$

Proof. Putting $P_{C} \equiv I$ in Corollary 7, we can obtain the desired conclusion immediately.

\section{Conflict of Interests}

The authors declare that there is no conflict of interests regarding the publication of this paper.

\section{Acknowledgments}

The first author was supported by the Thailand Research Fund and the King Mongkut's University of Technology Thonburi (Grant no. RSA5780059). The second author was supported by the Commission on Higher Education, the Thailand Research Fund, and Rajamangala University of Technology Lanna Chiangrai under Grant no. MRG5680157 during the preparation of this paper.

\section{References}

[1] P. Hartman and G. Stampacchia, "On some non-linear elliptic differential-functional equations," Acta Mathematica, vol. 115, pp. 271-310, 1966.

[2] W. A. Kirk, "A fixed point theorem for mappings which do not increase distances," The American Mathematical Monthly, vol. 72, pp. 1004-1006, 1965.

[3] P. L. Combettes, "A block-iterative surrogate constraint splitting method for quadratic signal recovery," IEEE Transactions on Signal Processing, vol. 51, no. 7, pp. 1771-1782, 2003.

[4] G. Gu, S. Wang, and Y. J. Cho, "Strong convergence algorithms for hierarchical fixed points problems and variational inequalities," Journal of Applied Mathematics, vol. 2011, Article ID 164978, 17 pages, 2011.

[5] S. A. Hirstoaga, "Iterative selection methods for common fixed point problems," Journal of Mathematical Analysis and Applications, vol. 324, no. 2, pp. 1020-1035, 2006.

[6] H. Iiduka and I. Yamada, "A subgradient-type method for the equilibrium problem over the fixed point set and its applications," Optimization, vol. 58, no. 2, pp. 251-261, 2009.

[7] G. Marino and H. K. Xu, "Explicit hierarchical fixed point approach to variational inequalities," Journal of Optimization Theory and Applications, vol. 149, no. 1, pp. 61-78, 2011.
[8] K. Slavakis and I. Yamada, "Robust wideband beamforming by the hybrid steepest descent method," IEEE Transactions on Signal Processing, vol. 55, no. 9, pp. 4511-4522, 2007.

[9] K. Slavakis, I. Yamada, and K. Sakaniwa, "Computation of symmetric positive definite Toeplitz matrices by the hybrid steepest descent method," Signal Processing, vol. 83, no. 5, pp. 1135-1140, 2003.

[10] I. Yamada, "The hybrid steepest descent method for the variational inequality problem over the intersection of fixed point sets of nonexpansive mappings," in Inherently Parallel Algorithms in Feasibility and Optimization and Their Applications, D. Butnariu, Y. Censor, and S. Reich, Eds., vol. 8, pp. 473-504, Elsevier, Amsterdam, Netherlands, 2001.

[11] Y. Yao, Y. J. Cho, and Y. Liou, "Iterative algorithms for hierarchical fixed points problems and variational inequalities," Mathematical and Computer Modelling, vol. 52, no. 9-10, pp. $1697-1705,2010$

[12] Y. Yao, Y. J. Cho, and Y.-C. Liou, "Hierarchical convergence of an implicit double-net algorithm for nonexpansive semigroups and variational inequality problems," Fixed Point Theory and Applications, vol. 2011, article 101, 2011.

[13] Y. Yao, Y. J. Cho, and P.-X. Yang, "An iterative algorithm for a hierarchical problem," Journal of Applied Mathematics, vol. 2012, Article ID 320421, 13 pages, 2012.

[14] Y. Yao, Y.-C. Liou, and C.-P. Chen, "Hierarchical convergence of a double-net algorithm for equilibrium problems and variational inequality problems," Fixed Point Theory and Applications, vol. 2010, Article ID 642584, 16 pages, 2010.

[15] I. Yamada, N. Ogura, and N. Shirakawa, "A numerically robust hybrid steepest descent method for the convexly constrained generalized inverse problems," in Inverse Problems, Image Analysis, and Medical Imaging, Z. Nashed and O. Scherzer, Eds., vol. 313 of Contemporary Mathematics, pp. 269-305, 2002.

[16] I. Yamada and N. Ogura, "Hybrid steepest descent method for variational inequality problem over the fixed point set of certain quasi-nonexpansive mappings," Numerical Functional Analysis and Optimization, vol. 25, no. 7-8, pp. 619-655, 2004.

[17] H. Iiduka, "Strong convergence for an iterative method for the triple-hierarchical constrained optimization problem," Nonlinear Analysis: Theory, Methods \& Applications, vol. 71, no. 12, pp. e1292-e1297, 2009.

[18] H. Iiduka, "Iterative algorithm for solving triple-hierarchical constrained optimization problem," Journal of Optimization Theory and Applications, vol. 148, no. 3, pp. 580-592, 2011.

[19] Y. Yao, Y.-C. Liou, and G. Marino, “Two-step iterative algorithms for hierarchical fixed point problems and variational inequality problems," Journal of Applied Mathematics and Computing, vol. 31, no. 1-2, pp. 433-445, 2009.

[20] Y. Yao, Y.-C. Liou, and S. M. Kang, "Algorithms construction for variational inequalities," Fixed Point Theory and Applications, vol. 2011, Article ID 794203, 12 pages, 2011.

[21] L.-C. Ceng, Q. H. Ansari, and J.-C. Yao, "Iterative methods for triple hierarchical variational inequalities in Hilbert spaces," Journal of Optimization Theory and Applications, vol. 151, no. 3, pp. 489-512, 2011.

[22] Z. Opial, "Weak convergence of the sequence of successive approximations for nonexpansive mappings," Bulletin of the American Mathematical Society, vol. 73, pp. 591-597, 1967.

[23] F. E. Browder, "Nonlinear operators and nonlinear equations of evolution in Banach spaces," Proceedings of Symposia in Pure Mathematics, vol. 18, pp. 78-81, 1976. 
[24] T. Suzuki, "Strong convergence of Krasnoselskii and Mann's type sequences for one-parameter nonexpansive semigroups without Bochner integrals," Journal of Mathematical Analysis and Applications, vol. 305, no. 1, pp. 227-239, 2005.

[25] H. K. Xu, "Iterative algorithms for nonlinear operators," Journal of the London Mathematical Society, vol. 66, no. 1, pp. 240-256, 2002. 


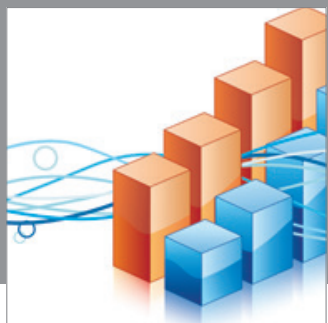

Advances in

Operations Research

mansans

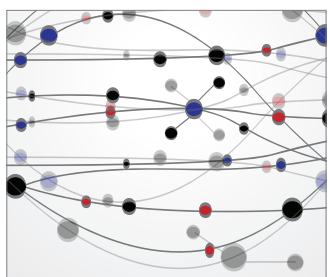

The Scientific World Journal
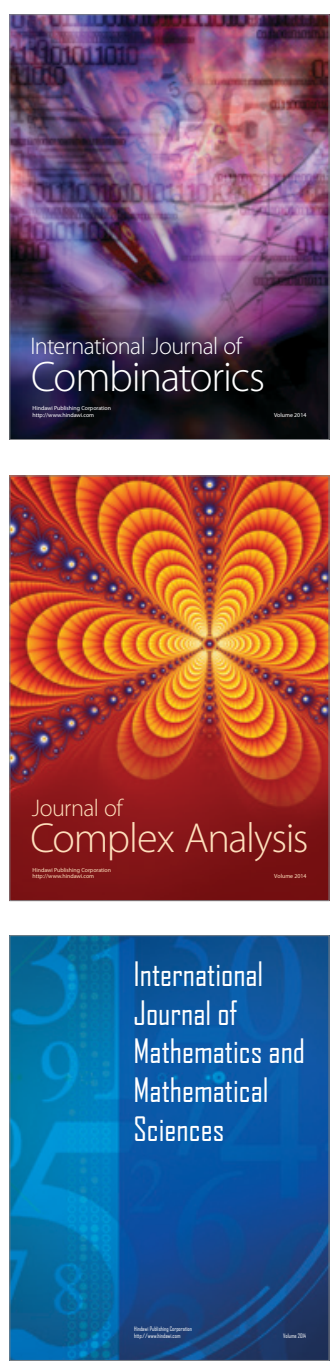
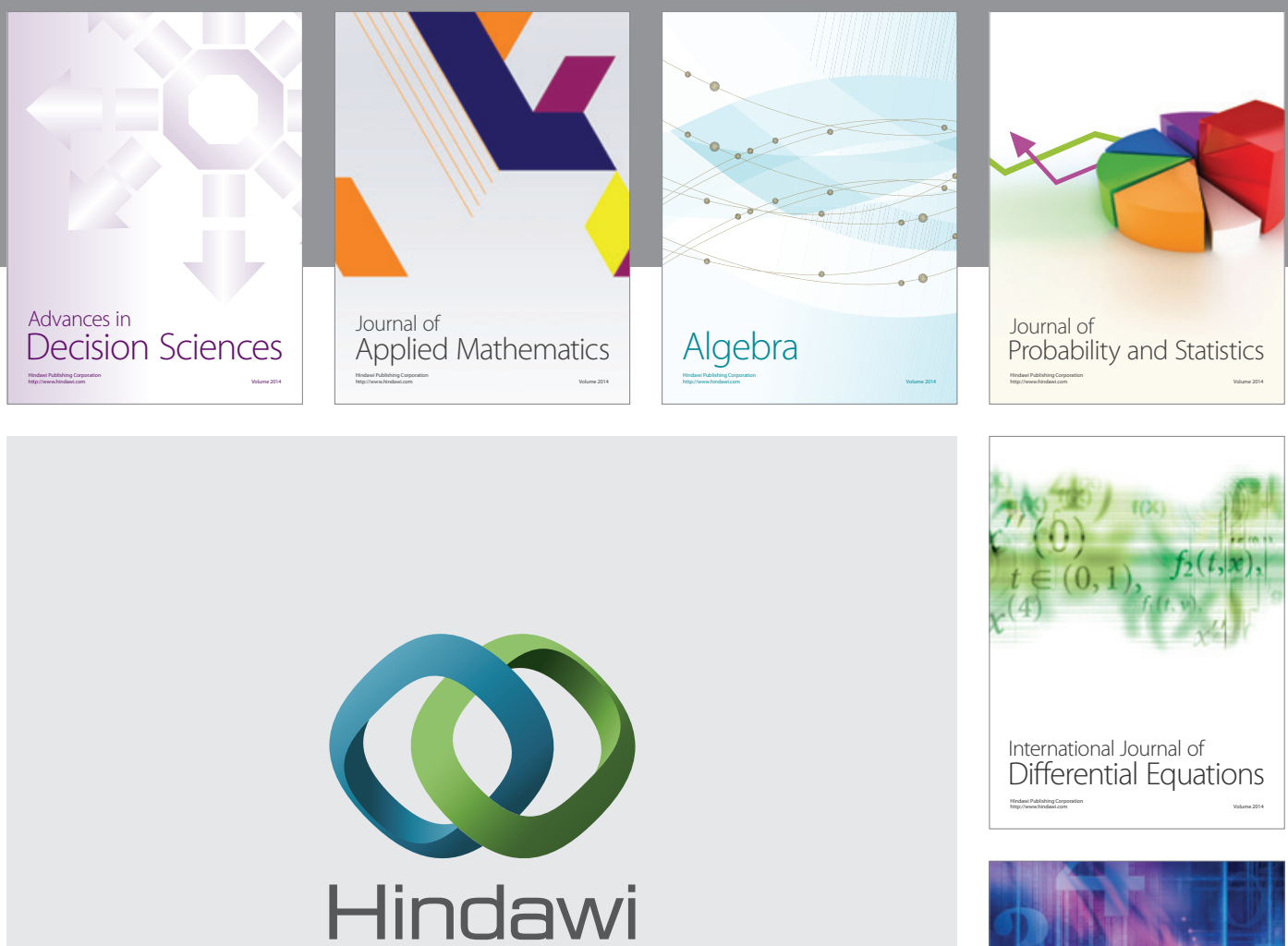

Submit your manuscripts at http://www.hindawi.com
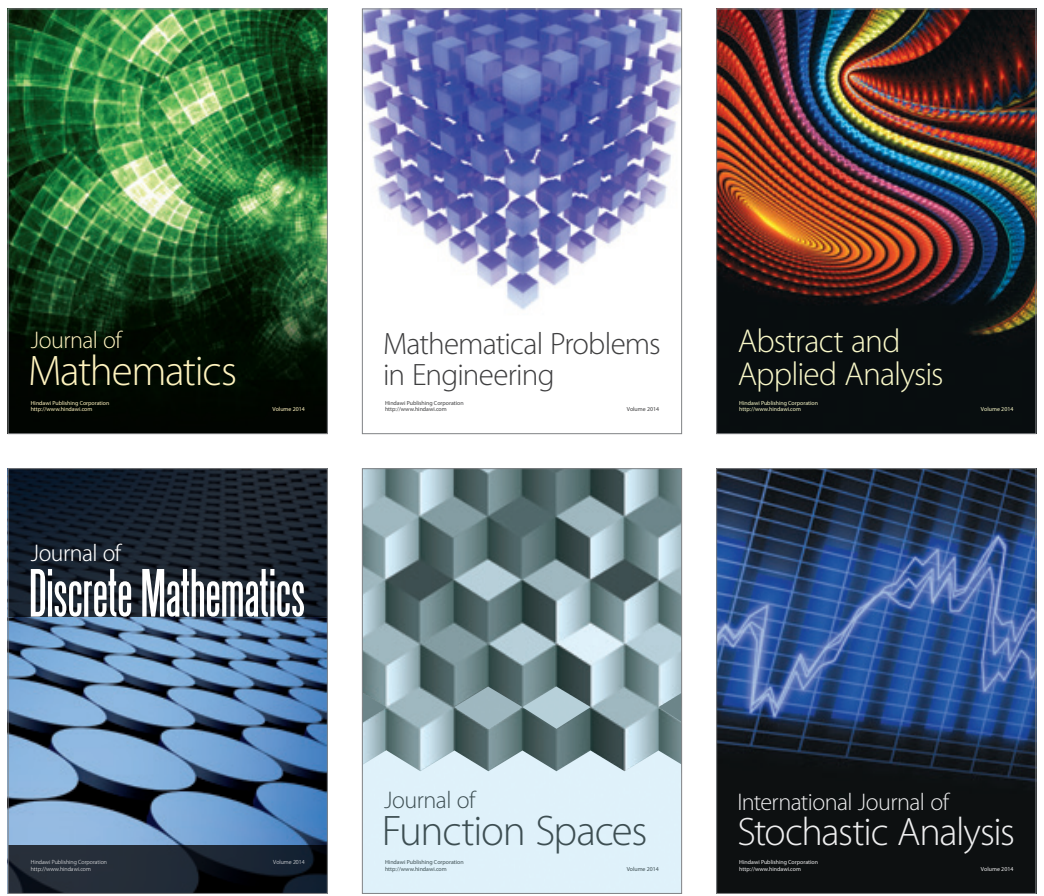

Journal of

Function Spaces

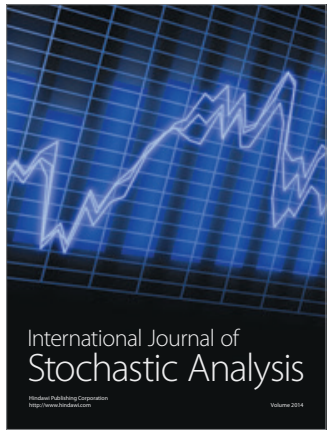

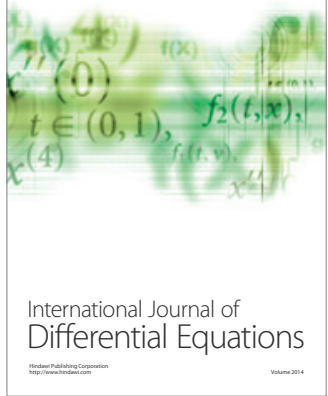
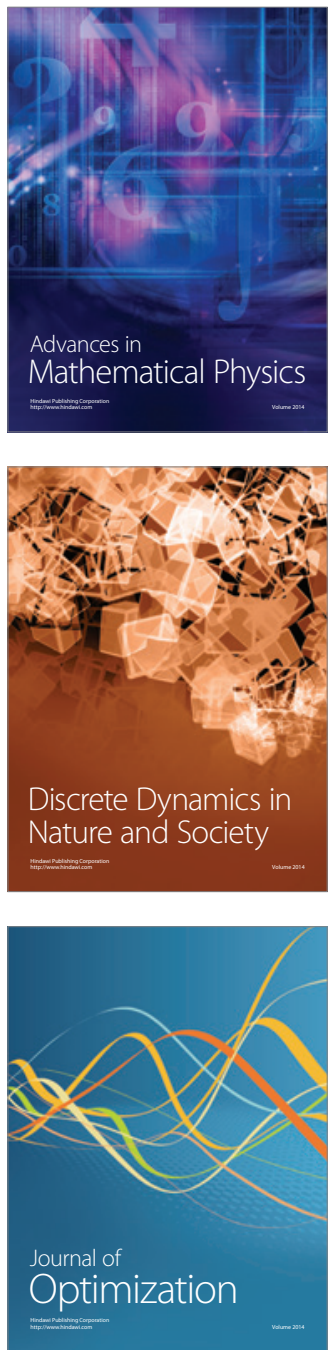